University of Nebraska - Lincoln

DigitalCommons@University of Nebraska - Lincoln

Entomology Papers from Other Sources

Entomology Collections, Miscellaneous

2010

Sugar in moderation: sugar diets affect short-term parasitoid behaviour

Danielle Lightle

Oregon State University

Mario Ambrosino

Oregon State University

Jana C. Lee

USDA-ARS

Follow this and additional works at: https://digitalcommons.unl.edu/entomologyother

Part of the Entomology Commons

Lightle, Danielle; Ambrosino, Mario; and Lee, Jana C., "Sugar in moderation: sugar diets affect short-term parasitoid behaviour" (2010). Entomology Papers from Other Sources. 18.

https://digitalcommons.unl.edu/entomologyother/18

This Article is brought to you for free and open access by the Entomology Collections, Miscellaneous at DigitalCommons@University of Nebraska - Lincoln. It has been accepted for inclusion in Entomology Papers from Other Sources by an authorized administrator of DigitalCommons@University of Nebraska - Lincoln. 


\title{
Sugar in moderation: sugar diets affect short-term parasitoid behaviour
}

\author{
DANIELLE LIGHTLE ${ }^{1}$, MARIO AMBROSINO ${ }^{2}$ and J A A C. LEE \\ ${ }^{1}$ Entomology Program, Oregon State University, Corvallis, Oregon, U.S.A., ${ }^{2}$ Integrated Plant Protection Center, Oregon State \\ University, Corvallis, Oregon, U.S.A. and ${ }^{3}$ USDA ARS, Horticultural Crops Research Unit, Corvallis, Oregon, U.S.A.
}

\begin{abstract}
The biological control potential of parasitic wasps in the field is expected to increase with the provisioning of sugar sources, which increase longevity and replenish carbohydrate reserves. Apanteles aristoteliae is an important parasitoid of Argyrotaenia franciscana, the orange tortrix, an economic pest in fruit crops. In the present study, the effect of sugar diet on the physiological status of A. aristoteliae is investigated in the laboratory, as well as the effects of nutritional status on short-term olfactory orientation and parasitism behaviour, and the association between olfactory orientation and immediate parasitism activity. Levels of glycogen, fructose, total sugars, proportional weight gain and volume consumed are higher among females fed $25 \%$ sucrose solution than $10 \%$ sucrose solution, and lowest for those fed water. Sugar feeding also affects behaviour: wasps with higher levels of fructose or weight gain have a lower probability of making a choice in the olfactometer. Wasps with intermediate fructose levels or weight gain have a higher probability of orienting towards hosts than wasps with low or high levels. Among wasps that make a choice in the olfactometer, wasps fed $10 \%$ or $25 \%$ sucrose significantly prefer host versus food cues, whereas starved wasps are just as likely to select food cues as host cues.
\end{abstract}

Key words. Apanteles aristoteliae, Argyrotaenia franciscana, olfactometer, oviposition, parasitoid, sugar, superparasitism.

\section{Introduction}

The benefits that sugars, in the form of aphid honeydew, nectar or sugar solutions, provide to parasitoid wasps are widely documented. Parasitoid wasps that consume sugar live significantly longer than individuals that are provided only with water (Wäckers et al., 2008). In addition to a longer lifespan, the lifelong fecundity of sugar fed wasps is higher (Jervis et al., 1996). The physiological state of a wasp, strongly influenced by diet, is predicted to affect the foraging behaviour of female parasitoids. The model of food and host foraging decisions of parasitoids by Sirot \& Bernstein (1996) predicts that when there is moderate to high food availability, parasitoids should forage for food before energy reserves become too low; but should not forage for food when food availability is very low. By contrast, the model of Tenhumberg et al. (2006)

Correspondence: Jana C. Lee, USDA ARS, Horticultural Crops Research Unit, 3420 NW Orchard Avenue, Corvallis, Oregon 97330, U.S.A. Tel.: +1 541738 4110; fax: +1 541738 4025; e-mail: jana.lee@ars.usda.gov predicts that parasitoids with low carbohydrate reserves should always search for food, no matter how scarce the food supply. Laboratory tests reveal that starved parasitoids ignore host cues in favour of food cues (Lewis \& Takasu, 1990; Jacob \& Evans, 2001).

Apanteles aristoteliae Viereck (Hymenoptera: Braconidae) is a solitary endoparasitoid of the leafroller pest known as the orange tortrix Argyrotaenia franciscana (Walsingham) (formerly Argyrotaenia citrana Fernald) (Lepidoptera: Tortricidae). Although this parasitoid is the dominant species attacking orange tortrix in caneberries, oranges, grapes and apples (Basinger, 1938; Kido et al., 1981; Coop et al., 1989; Walker \& Welter, 2004), the degree to which A. aristoteliae may be limited by insufficient nectar resources in these systems is unknown. Biological information on this species is lacking in the literature. This parasitoid does not appear to host-feed based on 4 years of experience with a laboratory colony; fieldcollected A. aristoteliae lived $2.92 \pm 0.37$ days with water $(n=13)$ and $32.0 \pm 1.8$ days with $25 \%$ honey $(n=51)(\mathrm{M}$. Ambrosino, personal observations). Therefore, this wasp may primarily extend its longevity via sugar feeding. 
The present study first determines how starvation and sugar feeding affect weight gain, sugar, glycogen and lipid levels of female A. aristoteliae. Second, the effects of diet and nutritional status on the short-term behaviour of the parasitoid in terms of olfactory preferences and reproductive output are investigated. Although many factors of feeding may affect activity, the present study examines how the amount of sugar consumed would affect behaviour while keeping the post-feeding time within a narrow range. Third, the relationship between olfactory preference and actual shortterm reproductive output is determined, particularly whether a preference for host odours indicates a greater likelihood to parasitize hosts.

\section{Materials and methods}

Behavioural tests were conducted with female A. aristoteliae that were $46-50-\mathrm{h}$ old; at this age, females readily orient towards host cues and engage in reproductive behaviours, as explained below. By this time, females can mature twice their egg load compared with emergence levels (33.3 \pm $1.0, n=122,17.1 \pm 1.4, n=31$, respectively) and would have many eggs available for a short-term trial on parasitism activity. Apanteles aristoteliae were reared on orange tortix in a laboratory culture maintained on diet at Oregon State University. Blackberry leaves (cultivar ORUS 1523-4 slated to be released commercially) used in behavioural assays were taken from plants grown in 3.8 -L pots in a greenhouse.

\section{Preparation}

First, females ( $0-4 \mathrm{~h}$ old) were paired with a male in a Petri dish $(100 \times 25 \mathrm{~mm})$ and a $10 \% \mathrm{w} / \mathrm{w}$ sucrose solution was provided in a $0.5-\mathrm{mL}$ microcentrifuge tube plugged with cotton (Table 1). Because sucrose is odourless, females were trained to associate feeding with a novel scent (Lewis \& Takasu, 1990; Patt et al., 1999) by adding $10 \mu \mathrm{L}$ of banana flavour (Kroger Co., Cincinnati, Ohio) in a $0.5-\mathrm{mL}$ microcentrifuge tube covered with mesh and attaching it to the sucrose tube. Petri dishes were kept in a growth chamber under a LD 16:8 h photocycle at $25{ }^{\circ} \mathrm{C}$ and $85 \%$ relative humidity. Females were allowed to feed ad libitum for $24 \mathrm{~h}$. Second, females were starved for the next $20.5 \mathrm{~h}$ and given a $0.5-\mathrm{mL}$ tube containing water. Also, during the starvation period, females (approximately $26 \mathrm{~h}$ old) were exposed to a leaf-host complex to condition them towards host cues. Conditioning was carried out because females reared from hosts fed artificial diet have a low probability of orienting towards host-plant cues in the olfactometer. Previously, $66 \%$ of females aged $24-48 \mathrm{~h}$ responded to host-plant cues after 5 min of conditioning on the previous day; whereas only $24 \%$ of naive unconditioned wasps responded ( $n=38$ ) (M. Ambrosino, personal observation). Females were placed in a Petri dish $(50 \times 9 \mathrm{~mm})$ with a host damaged blackberry leaflet and five second- to fourthinstar orange tortrix larvae, allowed to engage in host foraging and oviposition behaviour for $5 \mathrm{~min}$, and then females were returned to their original dish. Approximately $70 \%$ females would parasitize at least one larva in $5 \mathrm{~min}$. Third, after the starvation period, females were anesthetized with $\mathrm{CO}_{2}$ and weighed to $1 \mu \mathrm{g}$ on a Cahn C-35 microbalance (Thermo Electron Corp., Beverly, Massachusetts) to determine their prefeeding weight. Fourth, females were placed in a Petri dish with one of the food treatments: (i) water with no scent (starved treatment) $(n=46)$; (ii) $10 \%$ sucrose solution coupled with banana scent $(n=40)$; or (3) $25 \%$ sucrose solution coupled with banana scent $(n=40)$. Females had $1.5 \mathrm{~h}$ to feed and recover from any effects of the $\mathrm{CO}_{2}$ before the olfactometer trial began. Wasps were not weighed directly after the feeding period because preliminary trials showed that $\mathrm{CO}_{2}$ exposure affected how the wasps behaved in the olfactometer.

\section{Behaviour}

A Y-tube olfactometer (diameter $24 \mathrm{~mm}$ ) with carbon filtration (Analytical Research Systems, Micanopy, Florida) was used with $1.3 \mathrm{~L} \mathrm{~min}^{-1}$ airflow per arm with diffused lighting from above. Females were tested for their olfactory orientation towards food versus host cues. The food cue was $35 \mu \mathrm{L}$ of imitation banana flavour on a $50-\mathrm{mm}$ filter paper. Host cues were a host damaged blackberry leaflet, one host larva and frass. Each wasp was individually introduced into the olfactometer in a small glass couplet at the base of the Y-tube. Observations began when females crossed the start line $14 \mathrm{~cm}$ before the junction. A 'choice' for one of the cues was made when the wasp spent more than $15 \mathrm{~s}$ beyond the finish line, $6.5 \mathrm{~cm}$ after the junction in one of the olfactometer arms. Wasps that did not cross the start line or make a decision after $10 \mathrm{~min}$ were counted as 'no choice'. Preliminary trials suggested that naive wasps do not innately orient towards banana odour (three banana, one air control and 25 no choice),

Table 1. Sequence of procedures applied to individual female Apanteles aristoteliae.

\begin{tabular}{|c|c|c|}
\hline Duration (h) & Starting age $(\mathrm{h})$ & Procedures \\
\hline 24 & $0-4$ & o paired with $\sigma^{x}$, given $10 \%$ sucrose with banana odour ad libitum \\
\hline 20.5 & $26 \pm 2$ & o and $\sigma^{7}$ starved with water; approximately $26 \mathrm{~h}$ old $q$ exposed to host stimuli for $5 \mathrm{~min}$, host larvae frozen for dissection \\
\hline 0.2 & $46.5 \pm 2$ & o treated with $\mathrm{CO}_{2}$ and weighed (handling time included) \\
\hline 1.5 & $46.7 \pm 2$ & o given one of the treatments: (i) water; (ii) $10 \%$ sucrose with banana odor; and (iii) $25 \%$ sucrose with banana odour \\
\hline 0.2 & $48.2 \pm 2$ & o tested in olfactometer for orientation to host or food (banana) odours for $10 \mathrm{~min}$ (handling time included) \\
\hline \multirow[t]{2}{*}{1.5} & $48.4 \pm 2$ & o tested in parasitism arena, larvae frozen for dissection \\
\hline & $49.9 \pm 2$ & o treated with $\mathrm{CO}_{2}$, weighed, and frozen for ovary and nutrient analyses \\
\hline
\end{tabular}


and that trained and starved wasps orient towards banana (15, 0 and 11, respectively). As also mentioned previously, conditioned wasps could orient towards host-plant cues.

The same females were tested for their parasitism activity immediately after the olfactometer trial. An individual wasp was placed into the center of a clear plastic arena $(32 \times 25$ $\times 11 \mathrm{~cm}$ ). At one end of the arena was a blackberry leaf infested with 20 second- to fourth-instar orange tortrix larvae. Blackberry leaves had been infested for at least $24 \mathrm{~h}$ prior to the beginning of the trial. Females were allowed $1.5 \mathrm{~h}$ to move within the arena and host patch. The dimensions of the arena permitted females to fly, although they mostly walked. Afterwards, females were removed, weighed to determine the weight lost or gained from their last feeding and activity period, and frozen. Orange tortrix larvae used in the parasitism activity trial were also frozen.

\section{Analyses}

To determine the egg load, female wasps were dissected, their ovaries removed, and eggs counted. One forewing was removed and measured. Lipid, glycogen and sugar levels were determined in individual wasps using vanillin and anthrone in accordance with methods described by Olson et al. (2000). Thirty-one newly-emerged females were also dissected and tested for sugars and lipids for baseline data. All orange tortrix larvae exposed to the wasps were dissected for the presence of wasp eggs.

The effects of treatment on weight gain, volume gain (weight gain/weight of $1 \mu \mathrm{L}$ of $10 \%$ or $25 \%$ solution), lipid, glycogen, fructose, total sugar levels and egg load were tested using analysis of variance (ANOVA) or Kruskal-Wallis with chi-square approximation depending on the homogeneity of variances. Differences among treatments were tested with Tukey's honestly significant difference; or Wilcoxon pairwise analysis with a corrected $P$-value $\left(\alpha_{\text {new }}=1-(1-0.05)^{1 / \mathrm{k}}\right.$, where $k=$ pairwise comparisons; Sokal \& Rohlf, 1981). Preferences for odours in the olfactometer by treatment were tested using Pearson's chi-square analysis. Because each diet treatment creates wasps with a range of nutritional states, the direct effects of nutritional status (fructose level, weight change) on olfactometer orientation were determined by logistic regression. For comparison, raw data in the form of proportions for a given fructose and weight class are presented alongside the regressions. The effects of treatment on the number of eggs laid, the number of larvae parasitized with one or more eggs, and the number of larvae superparasitized in the arena were tested using ANOVA. In addition, ANOVA tested how choices in the olfactometer (no choice, host, food) translated into oviposition behaviour in the arena. The wing lengths of females were equal across treatments $(2.96 \pm 0.016 \mathrm{~mm})$, as were their pre-feeding weights $(1.29 \pm 0.023 \mathrm{mg})$ and the number of eggs laid during their conditioning period (1.07 \pm 0.85 ); thus, size and conditioning experience were not included in the analysis (winglength: $F_{2,119}=1.03, P=0.36$; pre-treatment weight: $F_{2,123}=0.651, P=0.523$; eggs laid in conditioning: $F_{2,122}=0.33, P=0.72$ ). Three females from the starved treatment escaped from the parasitism activity arena and were not assayed for nutrient levels, nor parasitism activity ( $n=43$ instead of 46). All statistics were conducted with JMP, version 7.0.1 (SAS Institute, 2007).

\section{Results and Discussion}

\section{Effects of diet on physiological status}

The study confirms that water, $10 \%$ and $25 \%$ sucrose diets affected female $A$. aristoteliae in terms of weight gain, volume of sucrose consumed and nutrient levels. There is a significant difference between all three treatments in the weight change that occurs during the 3.2-h feeding period and behaviour trial. Starved wasps lost $0.058 \pm 0.004 \mathrm{mg}$, whereas wasps fed $10 \%$ sucrose solution gained $0.099 \pm 0.017 \mathrm{mg}$ and those fed $25 \%$ sucrose solution gained significantly more weight at $0.18 \pm 0.019 \mathrm{mg}\left(\chi^{2}=77.8\right.$, d.f. $=2, P<0.001$; adjusted $P=0.017$ for pairwise comparisons). Wasps fed $10 \%$ sucrose consumed $0.123 \pm 0.012 \mu \mathrm{L}$, and wasps fed $25 \%$ sucrose consumed $0.166 \pm 0.017 \mu \mathrm{L}(n=72, F=4.2$, d.f. $=1$, $P=0.045)$. The tendency for $A$. aristoteliae to feed more from $25 \%$ than $10 \%$ sucrose is consistent with observations that diets with higher sugar concentrations lead to greater weight gain by Aphidius ervi (Azzouz et al., 2004) and elicit more gustatory responses among Anaphes iole and Diadegma semiclausum (Beach et al., 2003; Winkler et al., 2005).

The levels of fructose, total sugars and glycogen were significantly higher in sugar-fed than starved wasps, whereas the levels of total sugars and glycogen in starved wasps remained at emergence levels or below (Fig. 1). Elevated sugar and glycogen levels are consistently observed among other parasitic Hymenoptera and Diptera fed sugar diets (Olson et al., 2000; Lee et al., 2004; Fadamiro et al., 2005). The levels of sugars and glycogen in wasps starved for only $24 \mathrm{~h}$ suggests that $A$. aristoteliae females must feed once a day to survive in the field. This is supported by the observation that 15 females undergoing the 20.5-h starvation protocol did not survive until the first weighing period and were not tested.

Lipid levels followed a different trend from sugars because lipid levels were greater among newly-emerged females (Fig. 1). This is consistent with other evidence suggesting that parasitic wasps cannot create lipids de novo (Giron \& Casas, 2003; Visser \& Ellers, 2008). Unexpectedly, lipid levels of starved wasps were significantly higher than the lipid levels of wasps from either sugar treatment (Fig. 1). For other parasitoids, starved females exhibit a faster decline of lipid levels than females fed sugar ad libitum (Ellers, 1996; Lee et al., 2004; Fadamiro et al., 2005). Rather than providing sugar ad libitum, A. aristoteliae females were starved for $20.5 \mathrm{~h}$, given access to various diets for $1.5 \mathrm{~h}$, and then frozen $1.7 \mathrm{~h}$ later for nutrient analysis. It is unlikely that lipids were diverted to egg production because there were no differences in the egg load of females among treatments (see below). More studies are needed to confirm that lipids levels are lower among recently fed A. aristotliae and are not an artefact of this experimental design. 


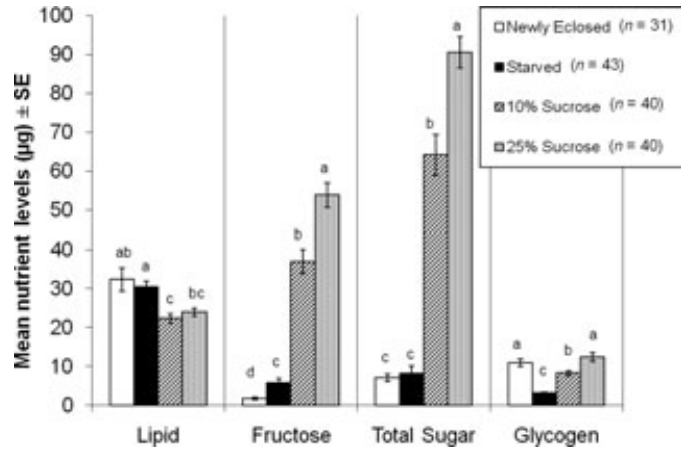

Fig. 1. Lipid and carbohydrate levels in female wasps that were newly emerged and in the three feeding treatments (lipid: $\chi^{2}=$ 19.0 , d.f. $=3, P<0.001$, mean rank of newly-eclosed wasps was lower than starved wasps; fructose: $\chi^{2}=114.8$, d.f. $=3, P<0.001$; total sugars: $\chi^{2}=110.5$, d.f. $=3, P<0.001$; glycogen: $\chi^{2}=81.9$, d.f. $=3, P<0.001)$. Letters denote significant differences between treatments using Wilcoxon pairwise analyses (adjusted $P=0.0085$ ).

Lastly, diet did not affect the number of mature eggs present in females when they were in the parasitism arena (eggs dissected from ovaries + eggs laid in arena, $F_{2,120}=$ $0.69, P=0.50)$. Starved wasps had $31.0 \pm 1.8$ eggs, whereas $10 \%$ and $25 \%$ sucrose fed wasps had $31.5 \pm 1.7$ and $33.8 \pm 1.9$ eggs, respectively. These results contrast with more eggs being found among Trichogramma nubilale fed sugar ad libitum (Olson \& Andow, 1998). However, the lack of difference may be expected because the present methods allowed little time for treatments to diverge: females were treated similarly until they were at least $44.7 \mathrm{~h}$ old and frozen for analysis $3.2 \mathrm{~h}$ later.

\section{Effects on olfactory orientation}

Diet treatment directly affected the three-way likelihood of a female choosing food, hosts or making no choice $\left(\chi^{2}=\right.$
22.1, d.f. $=4, P<0.001$; Fig. 2). Diet marginally affected the likelihood of females making a choice versus no choice $\left(\chi^{2}=5.1\right.$, d.f. $\left.=2, P=0.078\right)$. Approximately $39 \%$ (starved) to $60 \%$ (fed) of females in the olfactometer trials did not choose food, nor host odours (Fig. 2). Of the wasps that made a choice, starved wasps were equally likely to select food and host odours, whereas wasps fed $10 \%$ or $25 \%$ sucrose significantly preferred host odours (Fig. 2). Similarly, starved Cotesia rubecula are equally likely to choose host and food cues (Siekmann et al., 2004), whereas the preference for host cues among well-fed wasps is observed for $C$. rubecula (Wäckers, 1994; Siekmann et al., 2004), Microplitis croceipes (Lewis \& Takasu, 1990) and Bathylplectes curculionis (Jacob \& Evans, 2001).

The nutritional status of wasps regardless of diet treatment was examined for effects on behaviour. Fructose was used as indicator of feeding and gut sugar levels (Olson et al., 2000) because fructose is present at low levels $(1.74 \pm 0.37 \mu \mathrm{g})$ in newly-emerged unfed A. aristoteliae (Fig. 1) and sucrose breaks into glucose and fructose moieties. Fructose levels significantly associated with the choice females made in the olfactometer. Of wasps that made a choice in the olfactometer, females with levels of fructose lower than $12 \mu \mathrm{g}$ had a higher probability of selecting food cues, whereas females with levels above $12 \mu \mathrm{g}$ were more likely to select host cues (Fig. 3A). Also, the probability of making no decision in the olfactometer increased as fructose levels increased past $30-40 \mu \mathrm{g}$. Similarly, the proportion of wasps selecting food declines, whereas the proportion making no choice rises as fructose levels increase (Fig. 3B).

The proportion of weight gained or lost after the $3.2 \mathrm{~h}$ period was another indicator of feeding, and also had a significant effect on the olfactometer behaviour. Females that gained the most weight had a high likelihood of not making a choice (Fig. 3C). Logistic regressions followed trends similar to the proportional data, except that females that lost the most weight had a high probability of selecting food cues according to the regression (Fig. 3C), although the proportion of these

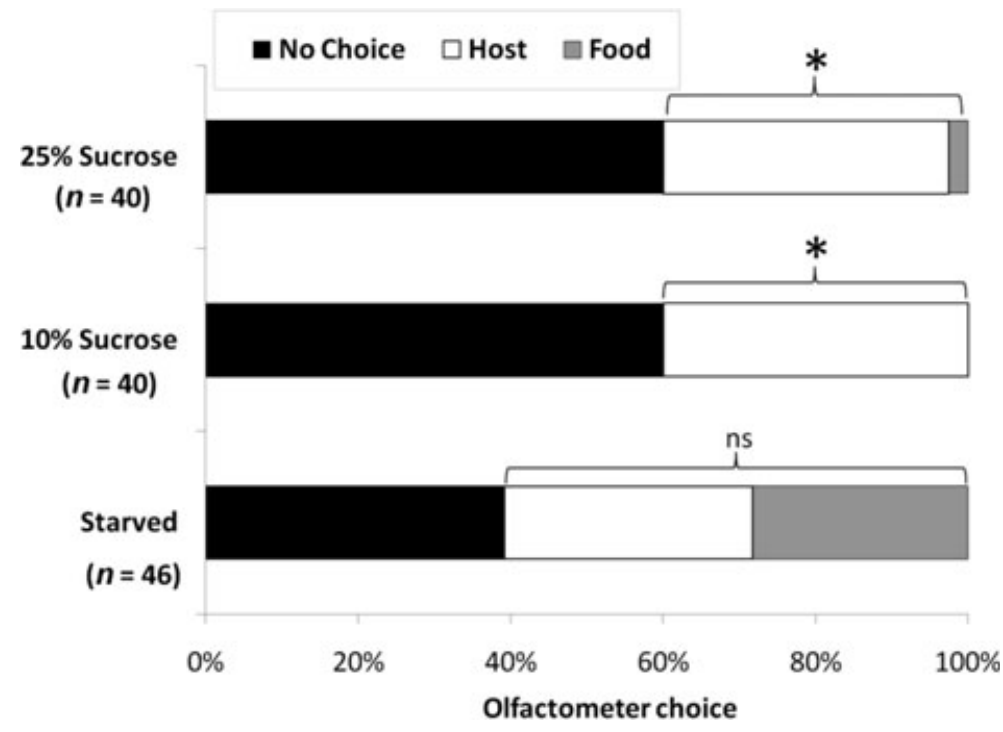

Fig. 2. Percentage of females that made no choice or oriented towards host or food cues. Among wasps that chose a cue, stars indicate a significant preference for an odour (starved: $\chi^{2}=0.14$, d.f. $=1, P=0.705$; $10 \%$ sucrose: $\chi^{2}=16$, d.f. $=1, P<0.001 ; 25 \%$ sucrose: $\chi^{2}=14.7$, d.f. $\left.=1, P<0.001\right)$. 
(A)

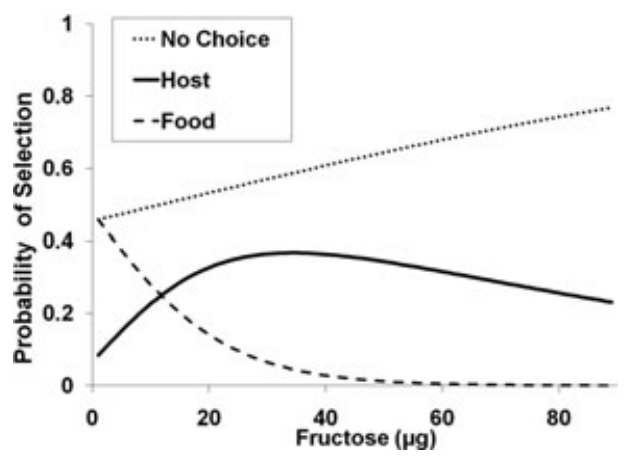

(C)

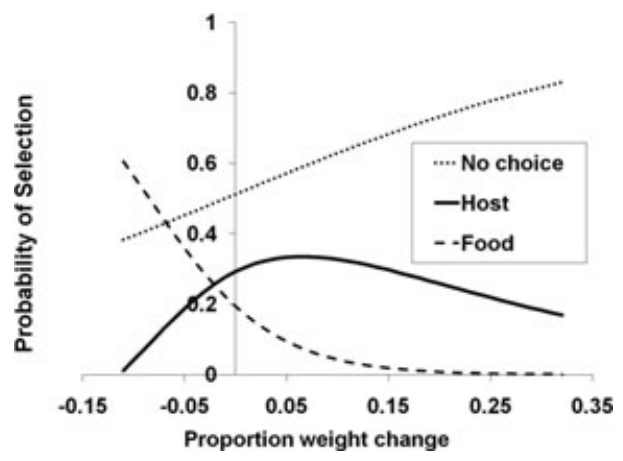

(B)

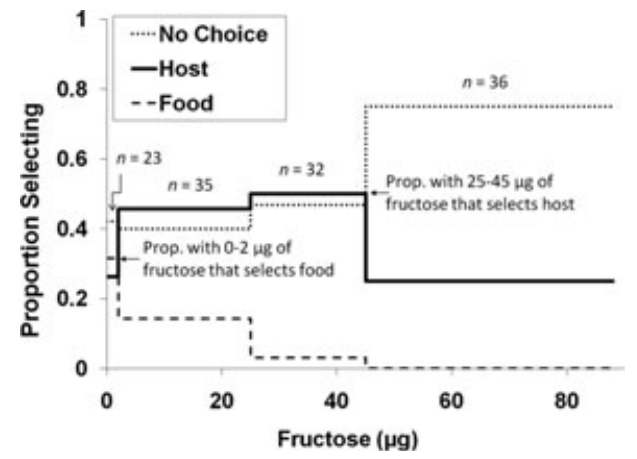

(D)

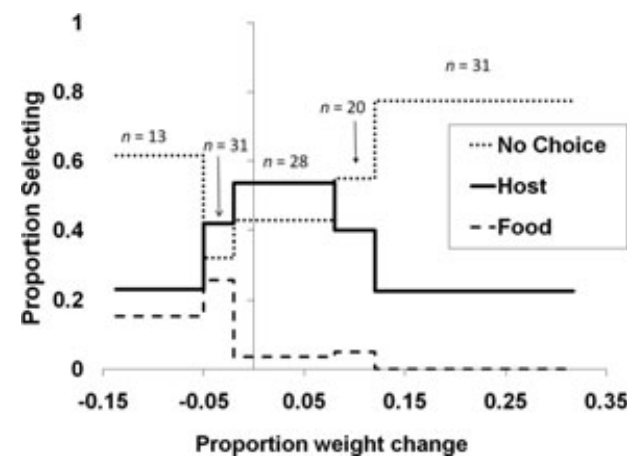

Fig. 3. (A) Probability of selecting either food cues, host cues or not making a choice in the olfactometer based on the gut fructose levels (logistic regression: $n=122, \chi^{2}=20.2$, d.f. $\left.=2, P<0.001, r^{2}=0.09\right)$. Probability food $=1-\{1 /[1+\exp (-0.084+-0.086 \times$ fructose $)]\}$; Probability $_{\text {no choice }}=1 /[1+\exp (0.18+-0.016 \times$ fructose $)]$; Probability host $=[1-($ Probablity no choice $)-($ Probability food $)]$. (B) Proportion of females within a fructose level category that selected food, host or made no choice. (C) Probability of and (D) proportion within a weight class selecting either food cues, host cues or not making a choice in the olfactometer based on the proportion of weight that females lost or gained (logistic regression: $n=123, \chi^{2}=19.0$, d.f. $\left.=2, P<0.001, r^{2}=0.08\right)$. Probability food $=1-\{1 /[1+\exp (-1.42+-16.82 \times$ proportion weight $\Delta)]\}$; Probability $_{\text {no choice }}=1 /[1+\exp (-0.050+-4.8 \times$ proportion weight $\Delta)]$.

females making no choice appeared common (Fig. 3D). Those wasps that consumed an intermediate amount of sugar with a proportional weight gain of $0.04-0.1$ were more likely to select host cues than wasps with substantial weight loss or gain. A period of inactivity is reported in female $D$. insulare fed sugar ad libitum, where females with higher levels of fructose are less likely to move into patches containing hosts (Lee \& Heimpel, 2007). Diadegma insulare are suggested to need a period of time to digest their sugar meal, accounting for the decreased probability of entering the patch. Similarly, A. aristoteliae may also need a period of time for digestion of their recent sugar meal, leading to the high probability of making no decision in the olfactometer trials when weight gain is substantial.

\section{Effect on parasitism}

Although treatment affected orientation in an olfactometer, there was no effect of treatment on the number of eggs laid, total larvae parasitized one or multiple times, and larvae superparasitized by wasps in the arena (Table 2). Alhough an abundance of hosts was available, A. aristoteliae superparasitized some hosts two to four times. The wasps were more than $46 \mathrm{~h}$ old when placed into the arena and had an egg load of more than 30 eggs, and it is unlikely that the lower rates of parasitism were observed because wasps had not matured enough eggs. It is possible that $A$. aristoteliae has a maximum number of eggs that can be laid in one patch. The single patches may have been left intentionally underexploited by wasps, even though they were still capable of ovipositing, to prevent self-superparasitism (Rosenheim \& Mangel, 1994). This possibility is likely given that $A$. aristoteliae on average laid $5.75 \pm 0.50$ eggs, still had $26.3 \pm 1.1$ eggs remaining in the ovaries, and did not appear to be capable of avoiding superparasitism because 5\% (i.e. one out of 20) larvae were superparasitized. Moreover, field studies of A. aristoteliae parasitism on patches with orange tortrix larvae show that the percentage of larvae parasitized remains approximately $40 \%$, regardless of the number of larvae in the patch (Walker \& Welter, 2004). In this case, treatment differences may appear only if wasps are monitored in larger multi-patch arenas.

Lastly, when examining the behaviour of females in the arena based on their choice in the olfactometer, there was a marginal association between olfactometer choice and the number of eggs laid, and for larvae superparasitized (Table 2). 
Table 2. Means \pm SE and analysis of variance on the effect of diet treatment and olfactometer choice on parasitism activity of Apanteles aristoteliae.

\begin{tabular}{llll}
\hline & Number of eggs laid & Number of larvae parasitized & Number of larvae superparasitized \\
\hline Starved & $4.65 \pm 0.83$ & $3.81 \pm 0.65$ & $0.70 \pm 0.22$ \\
$10 \%$ sucrose & $6.55 \pm 0.91$ & $5.0 \pm 0.65$ & $1.28 \pm 0.26$ \\
$25 \%$ sucrose & $6.10 \pm 0.88$ & $4.88 \pm 0.69$ & $1.12 \pm 0.22$ \\
d.f. & 2,120 & 2,120 & 2,120 \\
$F$ & 1.33 & 0.99 & 0.372 \\
$P$ & 0.269 & $3.86 \pm 0.49$ & 0.185 \\
No choice & $4.78 \pm 0.62$ & $5.57 \pm 0.63$ & $0.78 \pm 0.15$ \\
Host & $7.2 \pm 0.88$ & $4.33 \pm 1.42$ & $1.41 \pm 0.25$ \\
Food & $5.25 \pm 1.86$ & 2,120 & $0.83 \pm 0.51$ \\
d.f. & 2,120 & 2.24 & 2,120 \\
$F$ & 2.67 & 0.11 & 2.57 \\
$P$ & 0.073 & & 0.08 \\
\hline
\end{tabular}

Although comparisons were marginal, females that preferred host odours in the olfactometer later laid $44 \%$ more eggs in the arena compared with females that preferred food odours or that did not make a choice. Because A. aristoteliae does not host feed, the selection of host odours may indicate a preference for reproductive foraging. These observations are consistent with the assumption that the olfactory preferences of wasps in the olfactometer might reflect subsequent behavioural activity. The olfactory preferences of two-spotted spider mites Tetranychus urticae to host plants in the olfactometer are also consistent with their movement towards specific plants during a release-recapture study with different individuals in the greenhouse (Pallini et al., 1997). To the authors' knowledge, no other studies have correlated olfactory preference of the same individual parasitoid in an olfactometer to subsequent reproductive activity. In summary, A. aristoteliae with moderate sugar intake are more likely to orient to hosts in the short-term. Because a laboratory experiment demonstrates 'what parasitoids can do' and not necessarily 'what they actually do' in the field (Heimpel \& Casas, 2006), the present study demonstrates the need to explore how short-term effects of sugar intake can affect parasitoid foraging in the field.

\section{Acknowledgements}

We thank Mary Peterson and Chad Finn for providing blackberry plants; Paul Charron and Ted Mackey for assistance in keeping plants pest-free; and Dave Edwards and Joanna Fisher for their assistance with assays. We thank Erik Nelson for reviewing the manuscript. Funding was provided by USDA CRIS 5338-22000-032-00D.

\section{References}

Azzouz, H., Giordanengo, P., Wackers, F.L. \& Kaiser, L. (2004) Effects of feeding frequency and sugar concentration on behavior and longevity of the adult aphid parasitoid: Aphidius ervi (Haliday) (Hymenoptera: Braconidae). Biological Control, 31, 445-452.

Basinger, A.J. (1938) The orange tortrix. Argyrotaenia citrana. Hilgardia, 11, 635-669.
Beach, J.P., Williams, L., Hendrix, D.L. \& Price, L.D. (2003) Different food sources affect the gustatory response of Anaphes iole, an egg parasitoid of Lygus spp. Journal of Chemical Ecology, 29, $1203-1222$.

Coop, L.B., Knight, A. \& Fisher, G. (1989) Parasitism of orange tortrix on caneberry, Rubus spp, in western Oregon and Washington. Journal of the Entomological Society of British Columbia, $\mathbf{8 6}$, $63-65$.

Ellers, J. (1996) Fat and eggs: an alternative method to measure the trade-off between survival and reproduction in insect parasitoids. Netherlands Journal of Zoology, 46, 227-235.

Fadamiro, H.Y., Chen, L., Onagbola, E.O. \& Graham, L.F. (2005) Lifespan and patterns of accumulation and mobilization of nutrients in the sugar-fed phorid fly, Pseudacteon tricuspis. Physiological Entomology, 30, 212-224.

Giron, D. \& Casas, J. (2003) Lipogenesis in an adult parasitic wasp. Journal of Insect Physiology, 49, 141-147.

Heimpel, G.E. \& Casas, J. (2006) Parasitoid foraging and oviposition behavior in the field. Behavioral Ecology of Insect Parasitoids (ed. by E. Wajnberg, C. Bernstein and J. van Alphen), pp. 51-70. Blackwell Publishing, U.K.

Jacob, H.S. \& Evans, E.W. (2001) Influence of food deprivation on foraging decisions of the parasitoid Bathyplectes curculionis (Hymenoptera: Ichneumonidae). Annals of the Entomological Society of America, 94, 605-611.

Jervis, M.A., Kidd, N.A.C. \& Heimpel, G.E. (1996) Parasitoid adult feeding and biological control-a review. Biocontrol News and Information, $17,1 \mathrm{~N}-22 \mathrm{~N}$.

Kido, H., Flaherty, D.L., Kennett, C.E. et al. (1981) Seeking the reasons for differences in orange tortix infestations. California Agriculture, 35, 27-28.

Lee, J.C. \& Heimpel, G.E. (2007) Sugar feeding reduces shortterm activity of a parasitoid wasp. Physiological Entomology, 32, 99-103.

Lee, J.C., Heimpel, G.E. \& Leibee, G.L. (2004) Comparing floral nectar and aphid honeydew diets on the longevity and nutrient levels of a parasitoid wasp. Entomologia Experimentalis et Applicata, 111, 189-199.

Lewis, W.J. \& Takasu, K. (1990) Use of learned odours by a parasitic wasp in accordance with host and food needs. Nature, 348, 635-636.

Olson, D.M. \& Andow, D.A. (1998) Larval crowding and adult nutrition effects on longevity and fecundity of female Trichogramma nubilale Ertle \& Davis (Hymenoptera: Trichogrammatidae). Environmental Entomology, 27, 508-514. 
Olson, D.M., Fadamiro, H., Lundgren, J.G. \& Heimpel, G.E. (2000) Effects of sugar feeding on carbohydrate and lipid metabolism in a parasitoid wasp. Physiological Entomology, 25, 17-26.

Pallini, A., Janssen, A. \& Sabelis, M.W. (1997) Odour-mediated responses of phytophagous mites to conspecific and heterospecific competitors. Oecologia, 110, 179-185.

Patt, J.M., Hamilton, G.C. \& Lashomb, J.H. (1999) Responses of two parasitoid wasps to nectar odors as a function of experience. Entomologia Experimentalis et Applicata, 90, 1-8.

Rosenheim, J.A. \& Mangel, M. (1994) Patch-leaving rules for parasitoids with imperfect host discrimination. Ecological Entomology, 19, 374-380.

SAS (2007) JMP ${ }^{\circledR}$ Statistics and Graphics Guide, Version 7.0.1. SAS Institute, Cary, North Carolina.

Siekmann, G., Keller, M.A. \& Tenhumberg, B. (2004) The sweet tooth of adult parasitoid Cotesia rubecula: ignoring hosts for nectar? Journal of Insect Behavior, 17, 459-476.

Sirot, E. \& Bernstein, C. (1996) Time sharing between host searching and food searching in parasitoids: state-dependent optimal strategies. Behavioral Ecology, 7, 189-194.

Sokal, R.R. \& Rohlf, F.J. (1981) Biometry, 2nd edn. pp. 241-242. Freeman and Company, New York, New York.
Tenhumberg, B., Siekmann, G. \& Keller, M.A. (2006) Optimal time allocation in parasitic wasps searching for hosts and food. Oikos, 113, $121-131$.

Visser, B. \& Ellers, J. (2008) Lack of lipogenesis in parasitoids: a review of physiological mechanisms and evolutionary implications. Journal of Insect Physiology, 54, 1315-1322.

Wäckers, F.L. (1994) The effect of food deprivation on the innate visual and olfactory preferences in the parasitoid Cotesia rubecula. Journal of Insect Physiology, 40, 641-649.

Wäckers, F.L., van Rijn, P.C.J. \& Heimpel, G.E. (2008) Honeydew as a food source for natural enemies: making the best of a bad meal? Biological Control, 45, 176-184.

Walker, K.R. \& Welter, S.C. (2004) Biological control potential of Apanteles aristoteliae (Hymenoptera: Braconidae) on populations of Argyrotaenia citrana (Lepidoptera: Tortricidae) in California apple orchards. Environmental Entomology, 33, 1327-1334.

Winkler, K., Wäckers, F.L., Stingli, A. \& van Leteren, J.C. (2005) Plutella xylostella (diamondback moth) and its parasitoid Diadegma semiclausum show different gustatory and longevity responses to a range of nectar and honeydew sugars. Entomologia Experimentalis et Applicata, 115, 187-192.

Accepted 3 December 2009 